\title{
Outcome of Instrumental Oxygen Therapy in COVID-19: Survivors Versus Non-survivors in Bangladeshi Cohort
}

\author{
Morshed Nasir ${ }^{1, ~ *, ~ R a w s h a n ~ A r a ~ P e r v e e n ~}{ }^{1}$, Sonia Nasreen Ahmad ${ }^{2}$, Rumana Nazneen ${ }^{3}$, \\ Shafi Mohammad Parvez Ahmed ${ }^{4}$ \\ ${ }^{1}$ Department of Pharmacology, Holy Family Red Crescent Medical College, Dhaka, Bangladesh \\ ${ }^{2}$ Department of Medicine, Holy Family Red Crescent Medical College Hospital, Dhaka, Bangladesh \\ ${ }^{3}$ Department of Obstetrics and Gynaecology, Holy Family Red Crescent Medical College Hospital, Dhaka, Bangladesh \\ ${ }^{4}$ Department of Surgery, National Institute of Cardiovascular Diseases, Dhaka, Bangladesh
}

Email address:

morshednasir@hotmail.com (M. Nasir),morshed@hfrcmc.edu.bd (M. Nasir),rawshanperveen@gmail.com (R. A. Perveen), sonianasreen@gmail.com (S. N. Ahmad),rumana.nazneen@gmail.com (R. Nazneen), smparvezahmed27@gmail.com (S. M. P. Ahmed)

${ }^{*}$ Corresponding author

\section{To cite this article:}

Morshed Nasir, Rawshan Ara Perveen, Sonia Nasreen Ahmad, Rumana Nazneen, Shafi Mohammad Parvez Ahmed. Outcome of Instrumental Oxygen Therapy in COVID-19: Survivors Versus Non-survivors in Bangladeshi Cohort. American Journal of Internal Medicine.

Vol. 9, No. 1, 2021, pp. 52-57. doi: 10.11648/j.ajim.20210901.18

Received: January 27, 2021; Accepted: February 7, 2021; Published: February 23, 2021

\begin{abstract}
Background and objectives: Mortality of critically ill COVID-19 patients in ICU are high around the globe. There are variable reports on the outcome of invasive and non-invasive ventilation, change of oxygen saturation, and clinical characteristics in different countries and hospital set-ups. This study aimed to observe the demographic and clinical characteristics of critical COVID-19 cases, the trend of $\mathrm{SpO}_{2}$ in 10-days, and the mortality outcome of oxygen therapy in a tertiary level hospital in Bangladesh. Methods: In this retrospective study, data obtained from 99 patients admitted in ICU with COVID-19 was confirmed by RT-PCR of the nasopharyngeal swab. The 720-bed Holy Family Red Crescent Medical College Hospital (HFRCMCH), Dhaka, Bangladesh with a 9-bed ICU facility designated as "COVID-dedicated" from May17 to September 9, 2020. Ninety-nine patients were selected for the study, divided into two groups. 39 of them were non-survivors, whereas 60 included in the survivors group. Demographic data, correlation with age groups, clinical symptoms, instrumental oxygen therapy, and mortality were collected from hospital records. Appropriate statistical analysis was done using SPSS version 26.0. Results: Out of 99 patients admitted in ICU with COVID-19, 72 were male and 27 were female. The mean age of the patients was 61.08 years. Most of the ICU patients were in the 60-69 years of age group and the highest mortality rates $(35.89 \%)$ were observed in this age range. The presenting symptoms of the patients were shortness of breath $(85.85 \%)$ was the most common symptom followed by fever $(66.66 \%)$, cough (32.32\%), lethargy $(12.12 \%)$, and others $(7.77 \%)$. The mean $\mathrm{SpO}_{2}$ of their 10 -days ICU stay was also variable between the two groups. A gradual increase of mean $\mathrm{SpO}_{2}$ was observed in the survivors' group. Whereas, the mean $\mathrm{SpO}_{2}$ level of non-survivor had ups and downs from $92 \%$ to $83 \%$ on day-10, along with the lowest level of mean $\mathrm{SpO}_{2}(77 \%)$ was on the $7^{\text {th }}$ day. Conclusions: With the constrain of the healthcare support system and limited ICU facilities in a low-middle income country like Bangladesh, the mortality outcome and instrumental oxygen therapy to fight the ARDS caused by COVID-19 is far challenging. The present study clearly showed the highest mortality in patients who required mechanical ventilation, whereas, almost $75 \%$ of patients survived with high flow nasal cannula (HFNC). Therefore, the experience advocates the necessity of HFNC at the earliest possible time to avoid invasive ventilation in COVID-19 patients admitted in ICU.
\end{abstract}

Keywords: Mortality, COVID-19, Intensive Care, Mechanical Ventilation, Symptoms, Bangladesh 


\section{Introduction}

Severe acute respiratory syndrome (SARS) caused by a novel corona virus (CoV-2) was reported in Wuhan, China, in December 2019 that spread throughout the world [1]. COVID-19, the disease caused by the new corona virus, can cause lung complications such as pneumonia and, in the most severe cases, acute respiratory distress syndrome, or ARDS [2]. The possible complications of COVID-19 range from sepsis to long-lasting harm to the lungs, kidney, and other organs. Though most of the patients recover from pneumonia without lung damage, pneumonia associated with COVID-19 may be severe.

COVID-19 has become the unprecedented cause of death worldwide at present with an estimated around $5 \%$ of cases are critically ill, requiring intensive care unit (ICU) support [3]. The observed ICU mortality rate is highly variable in different countries for the pandemic. Initial reports from China and Italy have revealed overall mortality ranging from $26 \%$ to $62 \%$ and from Seattle and New York ranging from $23 \%$ to $50 \%$ in ICU [4]. Older age was a major predictor of mortality in studies from the United States, Italy, and China. The common clinical symptoms of patients in ICU were documented with fever, dry cough, shortness of breath, fatigue, and less commonly anosmia (loss of smell), dysgeusia (loss of taste), headache, and diarrhea [5].

Pneumonia associated with COVID-19 was reported as oxygen saturation $\left(\mathrm{SpO}_{2}\right)>90.5 \%$ predicted survival with a sensitivity of $84.6 \%$ in different studies in China and Europe that revealed a strong association between hypoxemia and mortality [6]. For some people, breathing problems can become severe enough to require treatment at the hospital with oxygen or even a ventilator. Pulmonary air sacs fill with fluid, limiting the ability to receive oxygen and causing shortness of breath, cough, and other symptoms. With the progression of COVID-19 pneumonia, fluid leaking from the tiny blood vessels in the lungs eventually leads to a form of lung failure, known as acute respiratory distress syndrome (ARDS). Patients with ARDS are often unable to breathe on their own and may require ventilator support to circulate oxygen in the body. People who survive ARDS and recover from COVID-19 may have lasting pulmonary scarring [7]. As there is no definitive pharmacotherapy proven against SARS-CoV2, remedesivir [8], favipiravir [9], tocilizumab, steroids, and convalescent plasma is also used in different countries to treat COVID-19. But the critical consideration of oxygen therapy is inevitable in almost every patient treated in ICU. As stated in the National Guideline on case management of COVID-19 in Bangladesh, there is no precise effective treatment for COVID-19, the main stay of management is early diagnosis and supportive care of symptoms and optimum support for organ function in severe illness [10]. No drug is yet recommended as chemoprophylaxis as there is no quality evidence of efficacy and safety in COVID-19.

Scientific research, observation, and sharing of experience are going on throughout the world to know more about this virus to combat critically ill patients requiring ICU. Though severe acute respiratory illness with fever and respiratory symptoms comprise the main clinical presentations, we must keep in mind the atypical presentations, so that no case remains undiagnosed. However, in the last two months, both the infection rate and death rate from COVID-19 have been escalating in the South Asian region, particularly in India, Pakistan, and Bangladesh. Moreover, not many studies have reported the ICU outcomes of critically ill COVID-19 patients with pulmonary involvement and ventilator interventions in Bangladesh since the outbreak. Understanding regional features are always important. There are few published studies on COVID-19 survivors and nonsurvivors requiring ICU support in hospitals dedicated to COVID-19 treatment in this country. So, we conducted this descriptive study on 99 critically ill COVID-19 cases admitted in the dedicated ICU of a tertiary care hospital to give a highlight on the demographic correlation, mortality outcome, the shift of oxygen saturation, and outcome of respiratory interventions in the Bangladeshi cohort.

\section{Methods}

The retro-prospective observational cohort study of 99 patients with COVID-19 was admitted to the ICU of Holy Family Red Crescent Medical College Hospital (HFRCMCH), Dhaka, Bangladesh from May 17 to September 9, 2020. The HFRCMCH was a 720-bed leading non-government hospital with a 9-bed ICU in the capital city designated as "COVID-dedicated" by the Government of Bangladesh for four months. Though the admission in to the ICU was at the discretion of the attending critical care physicians, the general criteria included all patients with confirmed COVID-19 (by RT-PCR of the nasopharyngeal swab) infection required increasing oxygen therapy. All consecutive patients admitted to the ICU in between the time frame were enrolled and 99 were conveniently selected as the study population. The study was approved by the designated hospital authority and the institutional ethics board (IERB/29/Res/Jul/2020/27/hf). We categorized age into six groups with 10 years' interval. Demographic data, correlation with age groups, clinical characteristics, instrumental oxygen therapy, percentage of oxygen saturation in 10-days' time, and mortality outcome were collected from hospital records. Statistical analysis (Chi-square test) was done using SPSS version 26.0 and all $\mathrm{P}$ values were two-tailed, with $\mathrm{P}<0.05$ considered statistically significant with a $95 \%$ confidence interval.

\section{Results}

Out of 99 patients admitted in ICU with COVID-19, 72 were male and 27 were female. The male: female ratio was 1:2.66 with an age range from 18 to 74 years. The mean age of the patients was $61.08( \pm 12.76)$ years. Most of the ICU patients were older males $(24.24 \%)$ and most of them were $60-69$ years of age group (34.34\%). The mean age of the 
survivors and non-survivors was $58.24( \pm 12.00)$ and 65.02 $( \pm 11.85)$ years respectively. Thirty-nine patients died $(39.39 \%)$, and sixty patients $(60.60 \%)$ were discharged alive from ICU, and the highest mortality rates were among patients 60 to 69 years old $(35.89 \%)$. There was a statistically highly significant difference $(\mathrm{P}<0.005)$ in mortality across the age groups (Table 1 and Figure 1).

The presenting symptoms of the patients were variable. Shortness of breath $(85.85 \%)$ was the most common symptom followed by fever (66.66\%), cough $(32.32 \%)$, lethargy $(12.12 \%)$, and others $(7.77 \%)$. Around $92.30 \%$
(36/39) of non-survivors had shortness of breath compared to $81.66 \%$ (49/60) survivors. Other symptoms include sore throat, myalgia, diarrhea, loss of taste, and none of the patients had a loss of smell in ICU (Table 2 and Figure 2).

The mean $\mathrm{SpO}_{2}$ of the 10-days outcome was also variable between survivors and non-survivors. Gradual and persistent increase of mean $\mathrm{SpO}_{2}$ was observed in survivors from day-1 (85\%) to day-10 (97\%). The mean $\mathrm{SpO}_{2}$ level declined from $92 \%$ to $83 \%$ on day-10. The lowest level of mean $\mathrm{SpO}_{2}(77 \%)$ was on the $7^{\text {th }}$ day among the non-survivors (Table 3 and Figure 3).

Table 1. Demographic profile of critical COVID-19 patients in ICU $(n=99)$.

\begin{tabular}{|c|c|c|c|c|c|c|c|c|c|}
\hline \multirow{2}{*}{ Age group (years) } & \multicolumn{4}{|c|}{ Survivors } & \multicolumn{4}{|c|}{ Non-survivors } & \multirow{2}{*}{ Chi-square } \\
\hline & male & female & total & Percentage & male & female & total & Percentage & \\
\hline Lessthan30 & 1 & - & 1 & $1.66 \%$ & - & - & - & - & \multirow{6}{*}{$\begin{array}{l}p \text {-value }=0.000356 \\
\text { Highly significant at } \\
p<0.005\end{array}$} \\
\hline 30-39years & 3 & 1 & 4 & $6.66 \%$ & 1 & 1 & 2 & $5.12 \%$ & \\
\hline 40-49years & 7 & 4 & 11 & $18.33 \%$ & 1 & - & 1 & $2.56 \%$ & \\
\hline $50-59$ years & 12 & 3 & 15 & $25.00 \%$ & 6 & 2 & 8 & $20.52 \%$ & \\
\hline 60-69years & 13 & 7 & 20 & $33.33 \%$ & 11 & 3 & 14 & $35.89 \%$ & \\
\hline 70andabove & 6 & 3 & 9 & $15.00 \%$ & 11 & 3 & 14 & $35.89 \%$ & \\
\hline
\end{tabular}
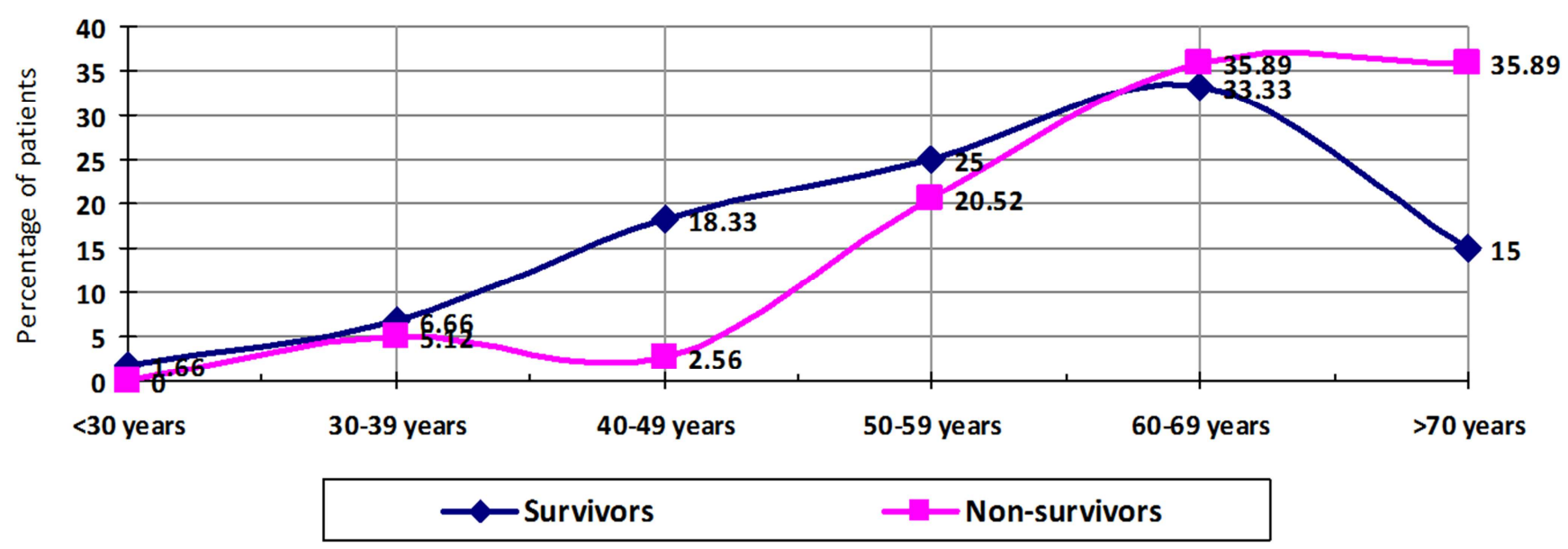

Figure 1. Distribution of survivor and non-survivor patients in ICU according to age groups.

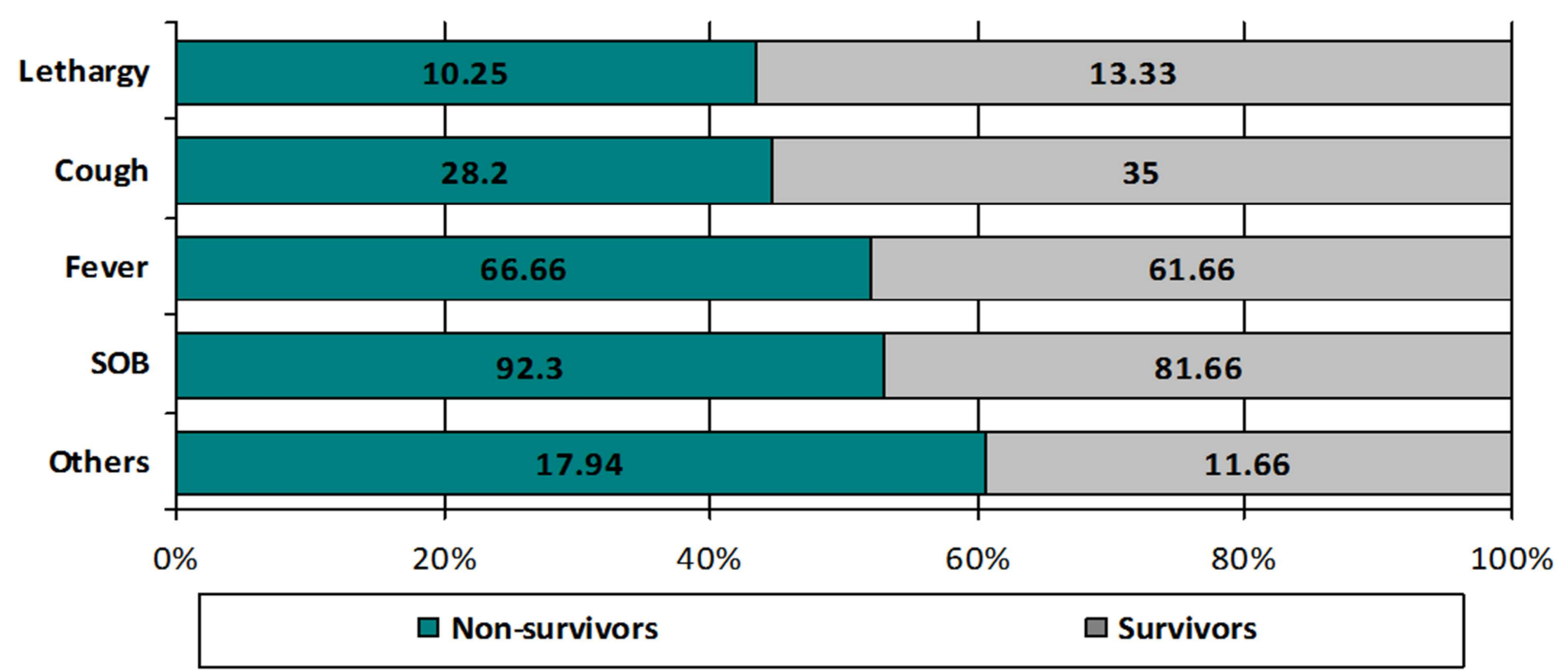

Figure 2. Symptoms of COVID patients in ICU among survivors and non-survivors. 
Table 2. Frequency of patients in ICU according to common clinical symptoms.

\begin{tabular}{lllllll}
\hline \multirow{2}{*}{ Total patients in ICU $(\mathbf{n}=\mathbf{9 9})$} & \multicolumn{2}{l}{ Survivors $(\mathbf{n}=\mathbf{6 0})$} & \multicolumn{2}{l}{ Non-survivors $(\mathbf{n}=\mathbf{3 9})$} & \multirow{2}{*}{ Statisticaloutcome } \\
\cline { 2 - 6 } & number & percentage & number & Percentage & \\
\hline \multirow{3}{*}{ Symptoms } & Fever & 37 & $61.66 \%$ & 26 & $66.66 \%$ & Chi-square \\
& Cough & 21 & $35.00 \%$ & 11 & $28.20 \%$ & 1.405 \\
& SOB & 49 & $81.66 \%$ & 36 & $92.30 \%$ & $p$-value=0.843 \\
\hline
\end{tabular}

Table 3. Change of mean $\mathrm{SpO}_{2}$ among the COVID-19 patients in 10-days outcome in ICU.

\begin{tabular}{llll}
\hline & Survivors $(\mathbf{n}=\mathbf{6 0})$ & Non-survivors $(\mathbf{n}=\mathbf{3 9})$ & Statistical outcome \\
\hline Day1 & $84.79 \pm 9.79$ & $82.68 \pm 11.73$ & \\
Day2 & $89.18 \pm 6.87$ & $83.70 \pm 13.56$ & \\
Day3 & $89.94 \pm 7.03$ & $83.04 \pm 12.34$ & Thet-valueis5.7737. \\
Day4 & $90.90 \pm 6.88$ & $79.80 \pm 17.84$ & The $p$-value is $<.00001$ \\
Day5 & $88.95 \pm 12.19$ & $82.86 \pm 14.83$ & Result is highly significant at $p<0.01$ \\
Day6 & $89.74 \pm 8.82$ & $84.09 \pm 10.76$ & \\
Day7 & $93.10 \pm 6.29$ & $77.75 \pm 29.98$ & \\
Day8 & $93.47 \pm 5.33$ & $86.75 \pm 5.5$ & \\
Day9 & $95.93 \pm 2.67$ & $86.33 \pm 2.89$ & \\
Day10 & $97.53 \pm 1.48$ & $82.67 \pm 4.16$ & \\
\hline
\end{tabular}

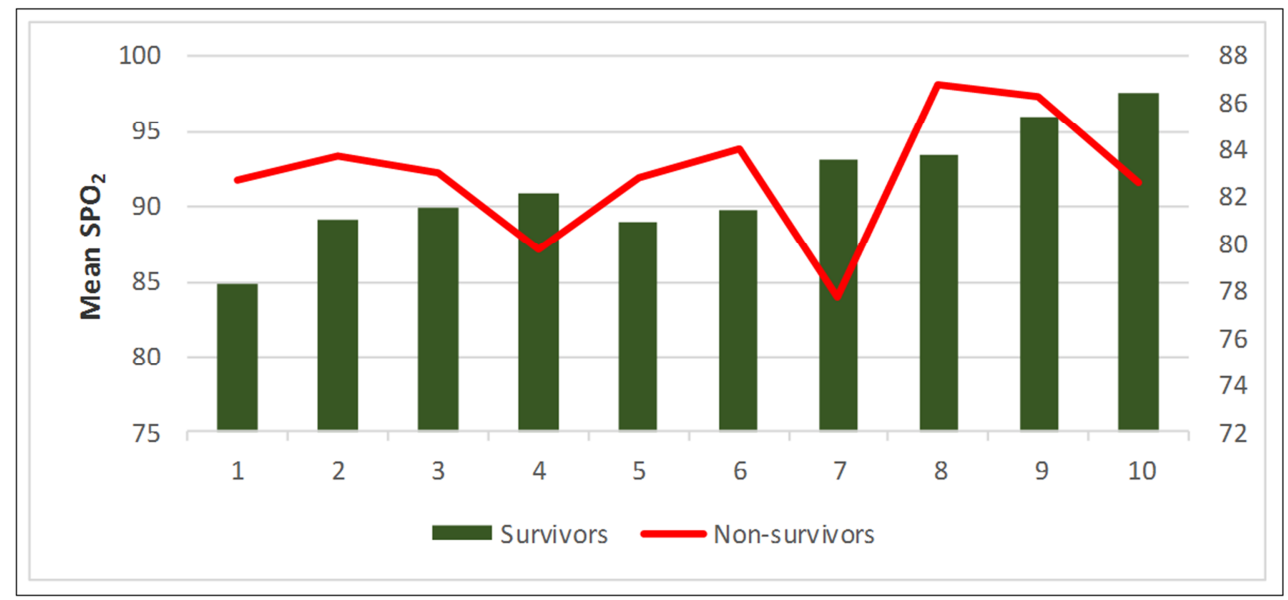

Figure 3. Mean $\mathrm{SpO}_{2}$ of 10-days outcome among survivors and non-survivors in ICU.

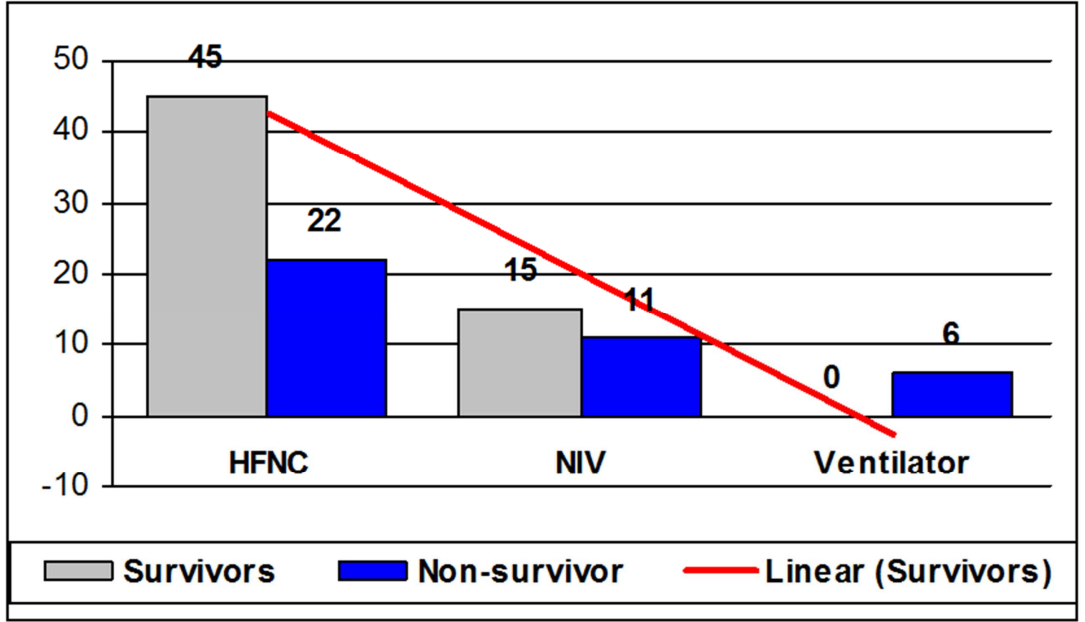

Figure 4. Outcome of instrumental oxygen therapy among survivors and non-survivors in ICU.

All of the 99 patients in ICU required instrumental oxygen therapy by high-flow nasal cannula (HFNC), non-invasive ventilator (NIV), and mechanical ventilators. Most of the patients $(68 / 99)$ patients were treated with HFNC and the mortality rate was $32.35 \%$. Whereas, a higher mortality rate was observed among patients with NIV (42.30\%). Only $6.06 \%$ 
of patients in ICU required mechanical ventilation and none of them could survive (Figure 4).

\section{Discussion}

The present study revealed the demographic data, correlation with age groups, clinical characteristics, requiring instrumental oxygen support, and mortality outcome of the COVID-19 patients during ICU stay. The mean percentage of oxygen saturation in the first ten days in critical COVID-19 patients was also recorded to observe the trend of severity.

The overall mortality rate in ICU was $39.39 \%$ in the present study, which was almost similar to the findings of King et al $(40 \%)$ [11]. The mortality rate of COVID-19 patients in ICU was almost similar in China, Italy, and Denmark with $37.7 \%, 25.6 \%$, and $41.2 \%$ respectively in different studies [12]. In a previous study on 58 patients in ICU, Nasir et al reported a $44.8 \%$ mortality rate ${ }^{5}$ and Hossain et al reported a high mortality rate of $76.2 \%$ in two different COVID-dedicated hospitals in Dhaka, Bangladesh [13]. In the UK, a study on 690 ICU admitted patients reported a $50.1 \%$ of mortality rate in ICU [14]. The heterogeneous variations in ICU mortality rate of COVID-19 patients raised continuous modification of management protocol in different countries.

The male dominancy (72.72\%) was observed among the ICU patients in this study and throughout the world. A study conducted in Mexico showed $66.3 \%$ of patients admitted in ICU were male [15]. In another study published about ICU admitted patients in Bangladesh observed $77.5 \%$ of patients were male [5] and death among males was more common than females [14]. In another observation, the severe group contains more than double (29) male patients than female (12) in ICU [16]. We observed a greater percentage of males in survivors $(42.42 \%)$ and non-survivors $(30.3 \%)$ than the females $(18.18 \%, 09.09 \%)$ in ICU.

Patients of advanced age account for the majority of death in the present study. A similar observation was reported in another study done on 164patients in the USA admitted in ICU, the highest $33 \%$ cases were seen in the 51-60 age group (survivors) and $38.6 \%$ cases in the above 70 -year group (nonsurvivors) [11]. In a Mexican study, 39.6\% of patients who were above 65 years of age were non-survivors, whereas survivors $(37.6 \%)$ were in the 35-54 age group [15]. Nasir et al reported in a study on 58 patients admitted in ICU, most of the critically ill patients were in the age range of 40-69 years [5]. But in another study in Bangladesh revealed that $28.6 \%$ of patients admitted in ICU were in the age range of 60-69 and more than 70 years [13]. Age and other general profiles of the patients may have influenced the prognosis of the patients. Data supports this concept that several thousand COVID-19 patients died in Europe and the USA, compared to Asian and African countries [17]. In a study in Bangladesh, the researcher found that patients who were dying due to COVID-19 had a significantly higher mean age than the alive group [16]. In the present study, the notable observation was that the highest percentage of patients were in the 60-69 years of age range among both survivors and the non- survivor $(35.89 \%, 33.33 \%)$.

The common symptoms of COVID-19 patients in ICU were almost similar in many studies. But the predominant symptoms vary in different populations. In the present study, the highest percentage of patients had shortness of breath among survivors (92.3\%) and non-survivor (81.66\%) in COVID-19 patients admitted in ICU followed by fever, cough, lethargy, and others (anosmia, myalgia, loss of taste, diarrhea, and sore throat). Other studies in Bangladesh also revealed similar findings with shortness of breath accounted for $92.1 \%$ [13]. In another study by Rawshan et al also reported shortness of breath as the commonest (86.42\%) symptom among 81 critical COVID-19 patients in ICU [19]. But a few studies have reported a lower number of patients with shortness of breath in ICU as $24 \%$ and $46 \%$ respectively [16-18]. The management of 'shortness of breath' as respiratory distress or ARDS is the main strategy in the treatment of COVID-19 patients in ICU.

No study has yet reported about the pattern and extent of change of mean $\mathrm{SpO}_{2}$ of patients in ICU from any hospitals in Bangladesh. In this study, we observed a change in their partial pressure of oxygen during the first 10-days of ICU stay. The trending line of the non-survivor group decline on the 4th, 7th, and 10th day among the non-survivors, whereas partial pressure of oxygen gradually increasing in the survivor group. There is a lack of clarity about the prognosis and intervention of COVID-19 patients with acute respiratory failure requiring invasive mechanical ventilation. The mortality rate ranges from $16 \%-97 \%$ in different clinical studies globally [11]. Studies in the USA reported $12.2 \%$ and $57.3 \%$ of patients in ICU required invasive mechanical ventilation [11, 19]. In Japan, a study on 1553 hospitalized COVID patients in 18 Japanese hospitals, 125 patients of which received invasive mechanical ventilation with a $24 \%$ mortality rate [20].

\section{Conclusion}

The present study clearly showed the highest mortality $(100 \%)$ in patients who required mechanical ventilation, whereas almost $75 \%$ of patients survived with high flow nasal cannula (HFNC) in COVID-19. Therefore, the COVID19 patients with ARDS should be recommended for HFNC at the earliest available time to avoid further deterioration and invasive ventilation in ICU. Early onset of non-invasive oxygen therapy might amend the outcome of invasive ventilation in ICU with limited facilities in low-middle income country like Bangladesh.

\section{Declarations of Interest}

None of the co-authors declared any potential competing interests include employment, consultancies, stock ownership, honoraria, paid expert testimony, patent applications / registrations, and grants or other funding. 


\section{Acknowledgements}

The authors acknowledge the dedication and cooperation of all healthcare workers and Mohammad Murshed, the Director of Holy Family Red Crescent Medical College Hospital, Dhaka, Bangladesh, during the study for obtaining data in the critical period.

\section{References}

[1] Zheng J. SARS-CoV-2: an Emerging Coronavirus that Causes a Global Threat. Int J Biol Sci. 2020 Mar 15; 16 (10): 16781685 doi: 10.7150/ijbs.45053. PMID: 32226285; PMCID: PMC 7098030.

[2] Panagis Galiatsatos. What Corona Virus Does to the Lungs. John Hopkins Medicine. Available at: https://www.hopkinsmedicine.org/health/conditions-anddiseases/coronavirus/what-coronavirus-does-to-the-lungs.

[3] Nadeem, A., Hamed, F., Saleh, K., Abduljawad, B. and Mallat, J. ICU outcomes of COVID-19 critically ill patients: An international comparative study. Anaesthesia Critical Care and Pain Medicine, 2020; 39 (4). https://doi.org/10.1016/j.accpm.2020.07.001.

[4] Vanderburg S, Alipanah N, Crowder R, Yoon C, Wang R, Thakur N, et al. Management and Outcomes of Critically-ill Patients with COVID-19 Pneumonia at a Safety-net Hospital in San Francisco, a Region with Early Public Health Interventions: A Case Series. medRxiv [Preprint]. 2020 May 29: 2020. $05.27 .20114090 . \quad$ doi: 10.1101/2020.05.27.20114090. PMID: 32511538; PMC 7273306 .

https://www.ncbi.nlm.nih.gov/pmc/articles/PMC7273306/.

[5] Nasir M, Perveen RA, Murshed $M$ et al. Survival and Biomarkers of COVID-19 Patients Treated with Remdesivir and Favipiravir in ICU: A Single Center Experience in Bangladesh, 08 December 2020, PREPRINT (Version1) available at Research Square https://doi.org/10.21203/rs.3.rs$123710 / \mathrm{v} 1$

[6] Jiang Xie, Naima Covassin, Zhengyang Fan, Prachi Singh, Wei Gao, Guangxi Li, et al Association Between Hypoxemia and Mortality in Patients With COVID-19. Mayo Clinic Proceedings, 2020; 95 (6): 1138-1147. https:// www.sciencedirect.com/science/article/abs/pii/S00256196203 03670 .

[7] Vasarmidi, E., Tsitoura, E., Spandidos, D. A., Tzanakis, N. and Antoniou, K. M. Pulmonary fibrosis in the after math of the Covid-19 era (Review). Experimental and Therapeutic

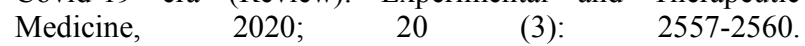
https://doi.org/10.3892/etm.2020.8980.

[8] Nasir M, Talha KA, Islam T, Saha SK, Selina F, Parveen RA. Use of Remdesivir in the Management of COVID-19: A Systematic Review on Current Evidences. Mymensingh Med J. 2020 Apr; 29 (2): 481-487. PMID: 32506110. https://pubmed.ncbi.nlm.nih.gov/32506110/.

[9] Nasir M, Perveen RA, Saha SK, Talha KA, Selina F, Islam MA. Systematic Review on Repurposing Use of Favipiravir Against SARS-CoV-2. Mymensingh Med J. 2020 Jul; 29 (3):
747-754. PMID:
https://pubmed.ncbi.nlm.nih.gov/32844821/.

32844821.

Nasir M, Chowdhury ASM, Zahan T. Self-medication during COVID-19 outbreak: A cross sectional online survey in Dhaka city. Int J Basic Clin Pharmacol. 2020 Sep; 9 (9): 1325-1330. DOI: 10.18203/2319-2003.ijbcp20203522. https://www.ijbcp.com/index.php/ijbcp/article/view/4308.

[11] King CS, Sahjwani D, Brown AW, Feroz S, Cameron P, Osborn E, et al. (2020) Outcomes of mechanically ventilated patients with COVID-19 associated respiratory failure. $\begin{array}{llll}\text { PLoSONE } & 15 & \text { (11): } & \text { e242651 }\end{array}$ https://doi:10.1371/journal.pone.0242651.

[12] Quah P, Li A, and Phua J, Mortality rates of patients with COVID-19 in the intensive care unit: a systematic review of the emerging literature. Crit Care. 2020; 24: 285. doi: 10.1186/s13054-020-03006-1PMID: 32498689 https://www.ncbi.nlm.nih.gov/pmc/articles/PMC7271132/.

[13] Hossain I, Khan MH, Tuhin SG, Aktaruzzaman MM, Rahman S, Mullick AR, Shahin M, et al. Baseline characteristics, level of disease severity and outcomes of patients with COVID-19 admitted to intensive care unit in COVID-19 dedicated Mugda Medical College and Hospital, Dhaka, Bangladesh. IJCMPH. 2020; 7 (10) DOI: http://dx.doi.org/10.18203/2394-6040.ijcmph20204347.

[14] Armstrong M. Covid-19 in the UK: intensive care survival rate. $7^{\text {th }}$ April, $2020 . \quad$ Available at: https://www.statista.com/chart/21360/uk-intensive-care-covid19-survival-rate/.

[15] Ñamendys-Silva SA. Healthcare workers with COVID-19 in Mexico. Eur Respir J 2020; IN PRESS. Availableat: https://doi.org/10.1183/13993003.02885-2020.

[16] Hossain MM, Mark SH, Kabir A, Das P, Islam MK, and Das A An Epidemiological Study of Laboratory Confirmed COVID19 Cases Admitted in Dhaka Medical College Hospital. J $\begin{array}{lllll}\text { Medicine. } & 2020 ; & 21 & \text { (2): } & \text { 69-75. }\end{array}$ https://doi.org/10.3329/jom.v21i2.50208.

[17] Bhuyan MA, Al Mahtab M, Ashab E, Haque MJ, Hoque SMM, Faizul Huq A, Islam MA, Choudhury N, Alia RA, Mahtab M, Khan MSI, Akbar SM. Treatment of COVID-19 Patients at a Medical College Hospital in Bangladesh. Euroasian J Hepatogastroenterol. 2020 Jan-Jun; 10 (1): 27-30. http://doi:10.5005/jp-journals-10018-1317 PMID: 32742969; PMCID: PMC7376594.

[18] Perveen RA, Nasir M, Murshed M, Naznin R, and Ahmed SN. Remdesivir and Favipiravir Changes Hepato-Renal Profile in COVID-19 patients: A Cross Sectional Observation in Bangladesh. IJMSCI, 2021; 8 (01): 5196-5201. https://doi.org/10.18535/ijmsci/v8i01.03.

[19] Walker M. High Mortality Rate in Intubated COVID-19 Patients in NYC - Comorbidities appeared to play a major role. Med Page Today. April 22, 2020 https://www.medpagetoday.com/infectiousdisease/covid19/86 10120.

[20] Hirayama A, Masui J, Murayama A et al. The characteristics and clinical course of patients with COVID-19 who received invasive mechanical ventilation in Osaka, Japan. IJID. 2021; 102: 282-284. https://doi.org/10.1016/j.ijid.2020.10.051. 